\title{
INTERNAÇÕES DE ADOLESCENTES POR CONDIÇÕES SENSÍVEIS À ATENÇÃO PRIMÁRIA EM UMA REGIONAL DE SAÚDE*
}

\author{
Jéssyca Slompo Freitas ${ }^{1}$, Maria Marta Nolasco Chaves ${ }^{2}$, Vivian Patricia Raksa ${ }^{3}$, Liliana Muller Larocca ${ }^{4}$
}

RESUMO: Objetivo: analisar as internações por condições sensíveis à Atenção Primária de adolescentes na segunda regional de saúde do Paraná no período de 2010 a 2014. Método: estudo ecológico retrospectivo, realizado no período de março a junho de 2016, com análise estatística descritiva de dados disponíveis no sistema de informações hospitalares. Resultados: das 87.321 internações de adolescentes de 10 a 19 anos, 9.858 $(11,29 \%)$ foram por condições sensíveis à atenção primária. Entre as principais causas: infecção do rim e trato urinário (23,28\%); gastroenterites infecciosas e complicações (18,96\%); epilepsias (14,91\%); doenças relacionadas ao pré-natal e parto (9,79\%); e asma (10,51\%). As internações na faixa etária de 15 a 19 anos representaram 57,52\% com prevalência para o sexo feminino $(66,64 \%)$. Conclusão: investigar a determinação das internações e dos processos que interferem na atenção primária à saúde para adolescentes pode reduzir hospitalizações evitáveis e aumentar a qualidade da atenção prestada ao adolescente.

DESCRITORES: Atenção primária à saúde; Hospitalização; Saúde pública; Saúde do adolescente; Enfermagem.

\section{HOSPITAL ADMISSIONS OF ADOLESCENTS DUE TO PRIMARY CARE SENSITIVE CONDITIONS IN A HEALTH REGION}

\begin{abstract}
Objective: To analyze hospital admissions due to primary care sensitive conditions in the 2nd Health Region of the State of Paraná in the 2010-2014 period. Method: Retrospective ecological study conducted from March to June 2016, with descriptive statistical analysis of data available in the hospital information system Results: Of the 87,321 admissions of adolescents aged 10-19 years, 9,858 (11.29\%) were due to primary care sensitive conditions. The main causes include kidney and urinary tract infection $(23.28 \%)$; infectious gastroenteritis and complications (18.96\%); epilepsies (14.91\%); diseases related to prenatal care and childbirth $(9.79 \%)$; and asthma (10.51\%). Hospitalizations in the age group of $15-19$ years accounted for $57.52 \%$, with a prevalence for the female gender $(66.64 \%)$. Conclusion: To investigate the determination of hospitalizations and processes that interfere with primary health care for adolescents can reduce preventable hospitalizations and increase the quality of care provided to adolescents.

DESCRIPTORS: Primary health care; Hospitalization; Public health; Adolescent health; Nursing.
\end{abstract}

\section{INGRESOS DE ADOLESCENTES POR CONDICIONES SENSIBLES A LA ATENCIÓN BÁSICA EN UNA REGIONAL DE SALUD}

RESUMEN: Objetivo: analizar los ingresos por condiciones sensibles a la Atención Básica de adolescentes en la segunda regional de salud de Paraná en el periodo de 2010 a 2014. Método: estudio ecológico retrospectivo, que se realizó en el periodo de marzo a junio de 2016, con análisis estadístico descriptivo de datos disponibles en el sistema de informaciones hospitalarias. Resultados: de los 87.321 ingresos de adolescentes de 10 a 19 años, 9.858 (11,29\%) ocurrieron por condiciones sensibles a la atención básica. Entre las principales causas, están: infección de los riñones y aparato urinario (23,28\%); gastroenteritis infecciosas y complicaciones $(18,96 \%)$; epilepsias (14,91\%); enfermedades asociadas al prenatal y parto $(9,79 \%)$; y asma (10,51\%). Los ingresos en la franja etaria de 15 a 19 años representaron 57,52\% con prevalencia para el sexo femenino (66,64\%). Conclusión: investigar la determinación de los ingresos y de los procesos que influyen en la atención básica a la salud para adolescentes puede reducir hospitalizaciones evitables y aumentar la calidad de la atención prestada al adolescente.

DESCRIPTORES: Atención básica a la salud; Hospitalización; Salud pública; Salud del adolescente; Enfermería.

*Artigo extraído da dissertação de mestrado “Internações por Condições Sensíveis à Atenção Primária de adolescentes na $2^{a}$ Regional de Saúde do Paraná". Universidade Federal do Paraná, 2016.

${ }^{1}$ Enfermeira. Mestre em Enfermagem. Universidade Federal do Paraná. Curitiba, PR, Brasil.

${ }^{2}$ Enfermeira. Doutora em Ciências. Docente do Programa de Pós-Graduação em Enfermagem da Universidade Federal do Paraná. Curitiba, PR, Brasil.

${ }^{3}$ Enfermeira. Mestre em Planejamento e Governança Pública. Enfermeira da Secretaria de Estado de Saúde do Paraná. Curitiba, PR, Brasil.

${ }^{4}$ Enfermeira. Doutora em Educação. Docente do Programa de Pós-Graduação em Enfermagem da Universidade Federal do Paraná. Curitiba, PR, Brasil. 


\section{INTRODUÇÃO}

A adolescência, período do desenvolvimento humano que compreende entre 10 e 19 anos de idade, merece destaque em discussões sobre suas concepções ${ }^{(1)}$, essencialmente as que estimulam direitos, deveres e oferecem subsídios para ações, organização do cuidado e políticas públicas voltadas a proteção do indivíduo nessa fase da vida ${ }^{(2)}$.

Nesta perspectiva, tem-se como desafio efetivar a diretriz doutrinária da integralidade, sendo este um dos princípios norteadores do Sistema Único de Saúde (SUS), definido como um conjunto articulado e contínuo das ações e serviços preventivos e curativos, individuais e coletivos, exigidos em todos os níveis de complexidade do sistema ${ }^{(3)}$.

Apesar de teoricamente o jovem ser considerado coparticipante na construção de políticas públicas no setor saúde, os programas têm pouca capacidade de propor mudanças e ações programáticas delineadas para os adolescentes, pois o foco dessas políticas continua voltado para riscos e vulnerabilidades ${ }^{(4)}$.

Atualmente, é preocupante a fragilidade do sistema na assistência à saúde de adolescentes, frente à exclusão das prioridades das gestões para efetivar políticas de saúde, uma vez que o sistema de assistência à saúde pode representar um dos elementos fundamentais na rede de suporte para as demandas deste grupo ${ }^{(5-6)}$. Diante disso, é evidente o fato de que o cuidado aos adolescentes na atenção básica se mostra com lacunas, entre as necessidades apontadas por eles e a efetivação de ações que os incluam como sujeitos que necessitam de cuidados específicos ${ }^{(7)}$.

No âmbito da atenção básica se percebe, ainda, a ausência de elementos organizacionais que envolvam os trabalhadores na atenção integral aos adolescentes, dificultando a resolubilidade dos problemas $^{(7)}$. Na perspectiva de nortear as políticas públicas e melhorar a atuação dos serviços, o desenvolvimento de indicadores de saúde é imprescindível à medida que contribui para a análise e organização do sistema de saúde ${ }^{(8)}$.

Indicadores passíveis de mensurar a efetividade da atenção primária, denominados de Internações por Condições Sensíveis à Atenção Primária (ICSAP), representam problemas de saúde que devem ser atendidos por ações do primeiro nível de atenção, e se não tratadas adequadamente, podem evoluir para uma hospitalização. Os indicadores também servem de instrumento para o monitoramento e a avaliação da atenção primária do sistema de saúde ${ }^{(9)}$.

No Brasil, após pesquisas nacionais e internacionais, reuniões com pesquisadores, gestores, especialistas e consulta pública, foi publicada pela Secretaria de Atenção à Saúde do Ministério da Saúde (SAS/MS) a lista brasileira de ICSAP, na forma de anexo da Portaria n. 221, de 17 de abril de 2008 ${ }^{(10)}$, composta por 19 grupos de diagnósticos conforme a Décima Revisão da Classificação Internacional de Doenças (CID-10) (Quadro 1). A prevenção de doenças, diagnóstico precoce, tratamento oportuno de patologias agudas, controle e acompanhamento de patologias crônicas, são exemplos de ações efetivas da atenção básica que resultariam em diminuição do número de $\operatorname{ICSAP}^{(8,10-11)}$.

Quadro 1 - Lista Brasileira de Condições Sensíveis à Atenção Primária. Brasil, 2008 (continua)

\begin{tabular}{|l|l|l|}
\hline Grupo & \multicolumn{1}{|c|}{ Diagnóstico } & \multicolumn{1}{c|}{ CID 10 } \\
\hline 1 & $\begin{array}{l}\text { Doenças preveníveis por imunização e } \\
\text { condições sensíveis }\end{array}$ & $\begin{array}{l}\text { A33-A37, A95, B05-B06, B16, B26, G00.0, A17.0, A19, } \\
\text { A15-A16, A18, A17.1-A17.9, I00-I02, A51-A53, B50-B54, B77 }\end{array}$ \\
\hline 2 & Gastroenterites Infecciosas e complicações & E86, A00-A09 \\
\hline 3 & Anemia & D50 \\
\hline 4 & Deficiências nutricionais & E40-E46, E50-E64 \\
\hline 5 & Infecções de ouvido, nariz e garganta & $\mathrm{H66,J00-} \mathrm{J03,} \mathrm{J06,} \mathrm{J31}$ \\
\hline 6 & Pneumonias bacterianas & $\mathrm{J} 13-\mathrm{J} 14, \mathrm{~J} 15.3-\mathrm{J} 15.4, \mathrm{~J} 15.8-\mathrm{J} 15.9, \mathrm{~J} 18.1$ \\
\hline 7 & Asma & $\mathrm{J} 45-\mathrm{J} 46$ \\
\hline 8 & Doenças pulmonares & $\mathrm{J} 20, \mathrm{~J} 21, \mathrm{~J} 40-\mathrm{J} 44, \mathrm{~J} 47$ \\
\hline
\end{tabular}


Cogitare Enferm. (23)4: e56188, 2018

\begin{tabular}{|l|l|l|}
\hline 9 & Hipertensão & $\mathrm{I} 10-\mathrm{I} 11$ \\
\hline 10 & Angina & $\mathrm{I} 20$ \\
\hline 11 & Insuficiência cardíaca & $\mathrm{I} 50, \mathrm{~J} 81$ \\
\hline 12 & Doenças cerebrovasculares & $\mathrm{I} 63-\mathrm{I} 67, \mathrm{I} 69, \mathrm{G} 45-\mathrm{G} 46$ \\
\hline 13 & Diabetes mellitus & $\mathrm{E} 10-\mathrm{E} 14$ \\
\hline 14 & Epilepsias & $\mathrm{G} 40-\mathrm{G} 41$ \\
\hline 15 & Infecção no rim e trato urinário & $\mathrm{N} 10-\mathrm{N} 12, \mathrm{~N} 30, \mathrm{~N} 34, \mathrm{~N} 39.0$ \\
\hline 16 & Infecção da pele e tecido subcutâneo & $\mathrm{A} 46, \mathrm{~L} 01-\mathrm{L} 04, \mathrm{~L} 08$ \\
\hline 17 & $\begin{array}{l}\text { Doença Inflamatória em órgãos pélvicos } \\
\text { femininos }\end{array}$ & $\mathrm{N} 70-\mathrm{N} 73, \mathrm{~N} 75-\mathrm{N} 76$ \\
\hline 18 & Úlcera gastrointestinal & $\mathrm{K} 25-\mathrm{K} 28, \mathrm{~K} 92.0, \mathrm{~K} 92.1, \mathrm{~K} 92.2$ \\
\hline 19 & Doenças relacionadas ao pré-natal e parto & $\mathrm{O} 23, \mathrm{~A} 50, \mathrm{P} 35.0$ \\
\hline
\end{tabular}

Fonte: Portaria n. 221, de 17 de abril de $2008^{(10)}$.

A lista brasileira de ICSAP pode ser usada para comparar o desempenho de diferentes serviços de saúde, avaliar os efeitos de políticas de saúde e como parte da avaliação da resolutividade, qualidade das ações e acessibilidade da atenção primária à saúde, como também fazer parte de investigações sobre iniquidades de acesso entre as regiões, comunidades e grupos populacionais ${ }^{(12)}$.

Sendo assim, considerando o indicador para a qualidade e acesso da população à Atenção Primária à Saúde, e ainda, a importância de garantir atenção integral e intersetorial aos adolescentes, este estudo teve como objetivo analisar as ICSAPde adolescentes na Segunda Regional de Saúde do Paraná no período de 2010 a 2014, com a finalidade de contribuir para a assistência a saúde deste grupo populacional.

\section{MÉTODO}

Estudo ecológico retrospectivo, que combina diferentes bases de dados a um número grande de pessoas $^{(13)}$, contribuindo para a caracterização da saúde em variações regionais, permitindo formular hipóteses a serem indagadas em futuros estudos ${ }^{(14)}$. A pesquisautilizou dados secundários para a descrição das Internações por Condições Sensíveis à Atenção Primária de adolescentes, de 10 a 19 anos, ocorridas na Segunda Regional de Saúde do Paraná, no período de 2010 a 2014.

Para coleta e interpretação dos dados, o estudo foi ancorado na Teoria da Intervenção Práxica em Enfermagem em Saúde Coletiva (TIPESC), a qual se constitui marco teórico e metodológico na intervenção da enfermagem na coletividade. A TIPESC propõe uma forma sistematizada para captar, interpretar e intervir no processo saúde-doença, por meio da realidade objetiva (RO) nas dimensões que a conformam: singular, particular e estrutural. Nestas dimensões se evidenciam as contradições entre elas e nelas próprias. Assim, busca-se compreender quais são os processos determinantes da $\mathrm{RO}$, sejam eles protetores ou de desgaste ${ }^{(15-16)}$. Este estudo utilizou as duas primeiras etapas do método, que consistem no conhecimento e interpretação da realidade objetiva de um dado fenômeno.

O estado do Paraná, segundo o censo demográfico de 2010, possuía 10.444.526 habitantes, distribuídos em quatro macrorregionais de saúde, 22 regionais de saúde e 399 municípios ${ }^{(17)}$. A regional de saúde deste estudo contava com uma população de 3.223.836 milhões de habitantes, concentrando, portanto, 30,9\% da população paranaense ${ }^{(18)}$. O total de adolescentes na faixa etária de 10 a 19 anos, 549.136, correspondia amais de $17 \%$ da população total da Regional.

Foram coletados dados de internação hospitalar de uma Regional de Saúde do Paraná, registrados no Sistema de Informações Hospitalares do SUS (SIH-SUS), disponíveis na página eletrônica que compõe o DATASUS- Departamento de Informática do SUS. O SIH-SUS possibilita avaliação e auditoria das instituições de saúde, públicas e conveniadas ao SUS, além de amparar a construção dos perfis de morbimortalidade hospitalar com intenção de avaliar a qualidade assistencial ofertada para a população ${ }^{(19)}$. Utilizou-se como auxílio o aplicativo Tabulador Oficial do Ministério da Saúde (TABWIN/ 
Datasus), versão Tabwin 3.2, para a seleção e descompactação dos registrosselecionados no SIH/SUS.

As condições sensíveis foram selecionadas a partir da lista de ICSAP, composta por 19 grupos de causas, que agrega as categorias da Classificação Internacional de Doenças (CID-10) ${ }^{(10)}$.Incluíram-se todas as Internações por Condições Sensíveis à Atenção Primária de adolescentes que ocorreram em municípios da Regional de Saúde de escolha, no período de 2010 a 2014. Não houve critérios de exclusão. Para melhor compreensão das internações dos adolescentes, as informações coletadas quanto ao sexo e a faixa etária foram subdivididas em grupos de 10 a 14 anos e 15 a 19 anos.

Os resultados foram apresentados por meio de tabelas e gráficos e sua análise por meio de estatística descritiva. Quanto aos aspectos éticos, não houve necessidade de autorização do Comitê de Ética em Pesquisa, por não envolver qualquer tipo de intervenção com seres humanos e por tratar-se de dados de domínio público.

\section{RESULTADOS}

Na Segunda Regionalde Saúde do Paraná, no período de 2010 a 2014, foram registradas 87.321 internações, exceto partos, de adolescentes na faixa etária de 10 a 19 anos, das quais 9.858 (11,29\%) ocorreram por Condições Sensíveis a Atenção Primária (CSAP).

Quanto às internações por grupos de CSAP, as cinco principais causas foram: Grupo 15 (Infecção no rim e trato urinário) com 2.295 (23,28\%) de internações; Grupo 2 (Gastroenterites Infecciosas e complicações) com 1.869 (18,96\%); Grupo 14 (Epilepsias) com 1.470 (14,91\%); Grupo 19 (Doenças relacionadas ao pré-natal e parto) com 965 (9,79\%); e, Grupo 7 (Asma) com 788 (7,99\%) internações sensíveis à atenção primária (Tabela 1).

Tabela 1 - Números e proporções de internação por condições sensíveis, segundo grupos de condições e sexo. Curitiba, PR, Brasil, 2010 a 2014

\begin{tabular}{lcccccc}
\multirow{2}{*}{$\begin{array}{l}\text { Grupos de Causas Sensíveis à Atenção Primária } \\
\text { (CSAP) }\end{array}$} & \multicolumn{2}{c}{ Feminino } & \multicolumn{2}{c}{ Masculino } & \multicolumn{2}{c}{ Total } \\
\cline { 2 - 7 } & $\mathbf{N}$ & $\mathbf{\%}$ & $\mathbf{N}$ & $\mathbf{\%}$ & $\mathbf{N}$ & $\%$ \\
\hline 1. Doenças prev. por imuniz. e condições sensíveis & 50 & 0,76 & 33 & 1 & 83 & 0,84 \\
\hline 2. Gastroenterites Infecciosas e complicações & 1.036 & 15,77 & 833 & 25,56 & 1869 & 18,96 \\
\hline 3. Anemia & 21 & 0,32 & 8 & 0,24 & 29 & 0,29 \\
\hline 4. Deficiências nutricionais & 57 & 0,87 & 51 & 1,56 & 108 & 1,09 \\
\hline 5. Infecções de ouvido, nariz e garganta & 55 & 0,84 & 66 & 2,01 & 121 & 1,22 \\
\hline 6. Pneumonias bacterianas & 75 & 1,14 & 92 & 2,8 & 167 & 1,69 \\
\hline 7. Asma & 411 & 6,26 & 377 & 11,46 & 788 & 7,99 \\
\hline 8. Doenças pulmonares & 121 & 1,84 & 113 & 3,44 & 234 & 2,37 \\
\hline 9. Hipertensão & 36 & 0,55 & 28 & 0,85 & 64 & 0,65 \\
\hline 10. Angina & 8 & 0,12 & 20 & 0,61 & 28 & 0,28 \\
\hline 11. Insuficiência cardíaca & 221 & 3,36 & 233 & 7,08 & 454 & 4,6 \\
\hline 12. Doenças cerebrovasculares & 36 & 0,55 & 59 & 1,79 & 95 & 0,96 \\
\hline 13. Diabetes mellitus & 298 & 4,54 & 245 & 7,45 & 543 & 5,5 \\
\hline 14. Epilepsias & 756 & 11,51 & 714 & 21,71 & 1470 & 14,91 \\
\hline 15. Infecção no rim e trato urinário & 2.111 & 32,14 & 184 & 5,59 & 2.295 & 23,28 \\
\hline 16. Infecção da pele e tecido subcutâneo & 132 & 2,01 & 186 & 5,65 & 318 & 3,22 \\
\hline 17. Doença Inflamatória órgãos pélvicos femininos & 153 & 2,33 & 0 & 0 & 153 & 1,55 \\
\hline 18. Úlcera gastrointestinal & 27 & 0,41 & 47 & 1,43 & 74 & 0,75 \\
\hline 19. Doenças relacionadas ao pré-natal e parto & 965 & 14,69 & 0 & 0 & 965 & 9,79 \\
\hline Total CSAP & 6569 & 66,64 & 3289 & 33,36 & 9858 & 100
\end{tabular}

Fonte: Brasil. Ministério da Saúde. DATASUS. Sistema de Informações Hospitalares do SUS. 
De acordo com a Tabela 1, com relação ao sexo, a análise das ICSAP, demonstrou que as meninas internam o dobro que os meninos, representando 6.569 (66,64\%) internações, se comparado ao sexo masculino que apresentou $3.289(33,36 \%)$ de ICSAP.

Analisando as ICSAP segundo faixa etária, os dados indicaram que as condições sensíveis impactaram principalmente os adolescentes entre 15 e 19 anos, pois do total de 9.858 internações, 5.671 (57,52\%) ocorreramnesta faixa etária, enquanto para os adolescentes de 10 a 14 anos essa proporção foi menor, com 4.187(42,48\%) de ICSAP (Tabela 2).

Tabela 2 - Números e proporções de internação por condições sensíveis, segundo grupos de condições e faixa etária. Curitiba, PR, Brasil, 2010 a 2014

\begin{tabular}{lcccccc} 
Grupos de Causas Sensíveis à Atenção Primária & \multicolumn{2}{c}{$\mathbf{1 0 - 1 4}$ anos } & $\mathbf{1 5 - 1 9}$ anos & \multicolumn{2}{c}{ Total } \\
\cline { 2 - 7 } (CSAP) & $\mathbf{N}$ & $\mathbf{\%}$ & $\mathbf{N}$ & $\mathbf{\%}$ & $\mathbf{N}$ & $\mathbf{\%}$ \\
\hline 1. Doenças prev. Por imuniz. E condições sensíveis & 20 & 0,48 & 63 & 1,11 & 83 & 0,84 \\
\hline 2. Gastroenterites Infecciosas e complicações & 1.126 & 26,9 & 743 & 13,1 & 1869 & 18,96 \\
\hline 3. Anemia & 9 & 0,21 & 20 & 0,35 & 29 & 0,29 \\
\hline 4. Deficiências nutricionais & 65 & 1,55 & 43 & 0,76 & 108 & 1,09 \\
\hline 5. Infecções de ouvido, nariz e garganta & 68 & 1,62 & 53 & 0,93 & 121 & 1,22 \\
\hline 6. Pneumonias bacterianas & 72 & 1,72 & 95 & 1,67 & 167 & 1,69 \\
\hline 7. Asma & 557 & 13,3 & 231 & 4,07 & 788 & 7,99 \\
\hline 8. Doenças pulmonares & 112 & 2,67 & 122 & 2,15 & 234 & 2,37 \\
\hline 9. Hipertensão & 39 & 0,93 & 25 & 0,44 & 64 & 0,65 \\
\hline 10. Angina & 0 & 0 & 28 & 0,49 & 28 & 0,28 \\
\hline 11. Insuficiência cardíaca & 269 & 6,42 & 185 & 3,26 & 454 & 4,6 \\
\hline 12. Doenças cerebrovasculares & 20 & 0,48 & 75 & 1,32 & 95 & 0,96 \\
\hline 13. Diabetes mellitus & 307 & 7,33 & 236 & 4,16 & 543 & 5,5 \\
\hline 14. Epilepsias & 933 & 22,28 & 537 & 9,47 & 1470 & 14,91 \\
\hline 15. Infecção no rim e trato urinário & 351 & 8,38 & 1.944 & 34,28 & 2295 & 23,28 \\
\hline 16. Infecção da pele e tecido subcutâneo & 158 & 3,77 & 160 & 2,82 & 318 & 3,22 \\
\hline 17. Doença Inflamatória órgãos pélvicos femininos & 17 & 0,41 & 136 & 2,4 & 153 & 1,55 \\
\hline 18. Úlcera gastrointestinal & 17 & 0,41 & 57 & 1 & 74 & 0,75 \\
\hline 19. Doenças relacionadas ao pré-natal e parto & 47 & 1,12 & 913 & 16,1 & 965 & 9,79 \\
\hline Total CSAP & 4187 & 57,52 & 5671 & 42,48 & 9858 & 100
\end{tabular}

Fonte: Brasil. Ministério da Saúde. DATASUS. Sistema de Informações Hospitalares do SUS.

Conforme os dados da Tabela 2, ao comparar os cinco grupos de ICSAP predominantes em cada subgrupo de faixa etária de adolescentes, há significativa semelhança entre as causas, sendo que os grupos de Gastroenterites Infecciosas e complicações, Epilepsias, Infecção no rim e trato urinário e Diabetes Mellitus se repetiram em ambos os subgrupos das faixas etárias.

\section{DISCUSSÃO}

Na Segunda Regional de Saúde do Paraná, a análise de ICSAP em adolescentes durante cinco anos, destacam-se que os cinco diagnósticos mais frequentes de ICSAP no cenário e população deste estudoconvergem com outras pesquisas realizadas no Brasil, com faixas etárias e recortes temporais semelhantes ${ }^{(20-21)}$.

Entendendo que o processo de adoecimento da população é determinado pela forma como os indivíduos estão inseridos na produção e, consequentemente, em uma sociedade que está estruturada, 
na reprodução social, por classes sociais ${ }^{(13,22)}$, percebeu-se que o delineamento dos grupos das ICSAP nesta Regional demonstrou, além do reflexo das diferenças de produção cultural, econômica e política em que os adolescentes estão inseridos, que as enfermidades ocorreram de maneira distinta entre sexos e suas diferentes faixas etárias.

Esta diferença permite refletir sobre a relevância de considerar, além das características biológicas destes indivíduos, fatores como a escolaridade, trabalho, renda familiar, desenvolvimento econômico da região, ou seja, as condições econômicas e sociais em que vivem para que se possa organizar a atenção ao adolescente, seja na promoção da saúde ou na prevenção de agravos.

Neste contexto, identifica-se a fragilidade de ações do setor saúde junto aos adolescentes, pois há invisibilidade do serviço de saúde para este segmento populacional, que aponta como orientadores para os cuidados que devem ter com seu corpoos amigos, familiares e a escola, não identificando o serviço de saúde como um vínculo ou aquele que promove a difusão de informaçõesde saúde ${ }^{(23)}$. De acordo com evidências e particularidades do estudo, constata-se que se houver investigação de internações desta população por condições sensíveis à atenção primária, refletir-se-á sobre a adoção de ações no nível primário de atenção à saúde para evitar tais internações.

Ao separar as ICSAP em adolescentes por sexo há maior frequência de hospitalizações em meninas, convergente com osresultados de outros estudos ${ }^{(24-26)}$. Acredita-se que há necessidade de se considerar a categoria de gênero na elaboração de políticas e ações de saúde para adolescentes, como uma possibilidade de reconhecer e enfrentar as desigualdades nas relações de gênero estabelecidas na sociedade, que acabam por determinar o processo saúde/doença dos sujeitos nessa fase da vida ${ }^{(27)}$. Atualmente, tal proposição tem sido comprovada em estudos epidemiológicos que estão aprofundando as discussões nessa temática, visto a complexidade das diferenças dessas relações na incidência de $\operatorname{agravos}^{(16)}$.

Neste sentido, ao refletir sobre os resultados deste estudo, registra-se a preocupação para além da questão anatômica, pois estesadolescentes são orientados para os seus cuidados de higiene, para vivenciar sua sexualidade e, consequentemente, terão autonomia para desenvolvê-los ao longo de suas vidas adultas, sem precisar conviver com internações recorrentes por uma causa previsível e com possibilidade de tratamento precoce. Porém, é necessário que tenham conhecimentos para serem capazes de reconhecer características e sinais de funcionamento do próprio corpo, no sentido de adotar iniciativas para um cuidado preventivo e busca de ajuda profissional para enfrentar o problema e suas necessidades em saúde.

De acordo com peculiaridades por faixa etária dos adolescentes, denotou-se que as ICSAP tiveram maior frequência na população com idade entre 15 a 19 anos. Neste aspecto, a atuação dos profissionais da atenção primária para o cuidado em saúde a este segmento populacional merece ser discutida. A educação em saúde, entre outras ações que visam à promoção da saúde, articuladas com outros setores presentes no território, devem buscar compreender o adolescente na sua integralidade e o estimular nas ações para os cuidados com a sua saúde. Considera-se que este princípio possibilita o fortalecimento de vínculo entre os profissionais e os usuários do serviço de saúde, bem como com a comunidade em geral.

Há necessidade de considerar as singularidades e especificidades dos adolescentes no avanço da organização dos serviços e na operacionalização de políticas públicas multissetoriais, pois se sobressai a diferenciação de comportamentos no que diz respeito aos aspectos psicossociais, sexuais e reprodutivos, sendo primordial expandir sua acessibilidade às ações preventivas e possibilitar sua participação nas instituições de saúde, além de garantir assistência de qualidade nas dimensões preconizadas pelo SUS ${ }^{(28)}$.

Relacionado ao aspecto de faixa etária, destaca-se a representatividade de adolescentes internados por Epilepsia, Asma e Diabetes Mellitus, haja vista que estas causas somaram $42,91 \%$ da proporção de internação sensível na faixa etária de 10 a 14 anos. Alguns aspectos são fundamentais no desafio de implementações de ações para os adolescentes, tais como contemplar a participação efetiva desses indivíduos na perspectiva das suas necessidades em saúde, onde a realidade local precisa ser constantemente problematizada, além de adotar uma organização em rede através de estratégias intersetoriais, educação permanente para o aperfeiçoamento da atuação dos profissionais, para um processo de trabalho articulado que vise à interdisciplinaridade para atendê-los ${ }^{(29)}$. 
Diante desses aspectos, percebe-se que, pelo fato de trazer indicação de possíveis problemas no acesso e na qualidade dos serviços de saúde, as ICSAP se constituem em um indicador importante não só para avaliação da atenção básica, como também para refletir sobre todo o sistema de saúde, uma vez que contribui para a discussão da concretização das diretrizes e dos princípios do SUS, pautados na integralidade, acessibilidade, universalidade, bem como a intersetorialidade ${ }^{(30)}$.

Ao refletir sobre os limites desta pesquisa, se destaca que o uso da lista brasileira de ICSAP, a qual é baseada na Classificação Internacional de Doenças (CID-10) e é de uso restrito dos profissionais médicos, restringe a prática assistencial e de investigação de acordo com as especificidades do processo saúde e doença dos adolescentes.

\section{CONCLUSÃO}

Este estudo sintetizou o panorama das Internações por Condições Sensíveis à Atenção Primária de adolescentes em uma Regional de Saúde do Paraná, ocorridas no período de 2010 a 2014, acentuando as relações por faixa etária, sexo e grupos de causas mais evidentes.

Destaca-seque os adolescentes estão sendo internados por CSAP, apesar do número destas internações serem pequeno, se comparado às demais faixas etárias internadas por CSAP. Porém, estas internações merecem investigação mais aprofundada, visto a importância de se considerar à dificuldade de efetivaras ações de promoção da saúde e prevenção de agravos, as quais devem estar ancoradas nos princípios da integralidade e resolubilidade para os adolescentes moradores das áreas de abrangência de serviços locais.

Nesta perspectiva, visando uniformização de registros dos diagnósticos e das intervenções, sugere-se que ações dos enfermeiros na Atenção Primária a Saúde (APS) sejam registradas com base na Classificação Internacional das Práticas de Enfermagem em Saúde Coletiva (CIPESC ${ }^{\circledast}$ ), pois esta representa uma possibilidade de instrumentalizar a prática profissional, a partir de registros que identifiquemo processo saúde-doença pelos diagnósticos de enfermagem dos adolescentes que utilizam esses serviços de saúde local, assim como intervenções que foram desenvolvidas junto ao adolescente.

Um banco de dados sobre os atendimentos dos enfermeiros junto aos adolescentes na APS permitiria o desenvolvimento de estudos que confrontassem essas informações e evidenciassem o acesso desse grupo populacional às ações disponibilizadas pelos serviços, assim como, permitiria reflexões sobre a resolutividade das ações dos diferentes profissionais da equipe.

Posteriormente, essas discussões poderiam ser subsídios para as reflexões mais aprofundadas sobre as ICSAP de adolescentes. Além disso, seria necessário que, nos processos de territorialização dos serviços locais de saúde, esse grupo populacional fosse descrito com base nas especificidades do modo de viver, pois a partir desta descrição haveriasubsídios para uma discussão de intervenções intersetoriais, que promovessem ações mais resolutivas frente às condições de vida dos adolescentes.

Considera-se que as ICSAP podem auxiliar no conhecimento acerca da saúde do adolescente na APS, conforme as reflexões já apresentadas, porém, ressalta-se que os processos que determinam a ida dos indivíduos aos serviços de saúde têm relação com processos sociais, econômicos e culturais. Portanto, conhecer a determinação do processo saúde-doença dos adolescentes nas dimensões particular e estrutural, os quais conformam a realidade observada - dimensão singular -, poderá fundamentar a elaboração de intervenções que objetivem a modificação das realidades de saúde encontradas nessa população, e assim, permitiriam refletir sobre intervenções intersetoriais que atendessem às necessidades de adolescentes.

Conclui-se que nos resultados e discussões deste estudo foi destacadaa importância do planejamento de ações da atenção primária, individual e coletiva, para adolescentes frente às suas especificidades, no sentido de promover a saúde desses indivíduos, assim como prevenir os agravos que os levaram a internações identificadas nesta pesquisa. É com este compromisso que se acredita iniciar um processo de discussão para a redução dos índices de ICSAP e melhora da qualidade da atenção à saúde dos adolescentes. 
1. Ministério da Saúde (BR). Secretaria de Atenção à Saúde Departamento de Ações Programáticas Estratégicas. Diretrizes nacionais para a atenção integral a saúde de adolescentes e jovens na promoção, proteção e recuperação da saúde [Internet] Brasília: Ministério da Saúde; 2010 [acesso em 2016 ago 24]. Disponível: http://bvsms.saude. gov.br/bvs/publicacoes/diretrizes_nacionais_atencao_saude_adolescentes_jovens_promocao_saude.pdf.

2. Silva CR, Lopes RE. Adolescência e juventude: entre conceitos e políticas públicas. Cad. Ter. Ocup. UFSCar [Internet]. 2009 [acesso em 2015 ago 29]; 17(2). Disponível em: http://www.cadernosdeto.ufscar.br/index.php/ cadernos/article/viewFile/100/65.

3. Ayres JRCM, Carvalho YM, Nasser MA, Saltão RM, Mendes VM. Caminhos da integralidade: adolescentes e jovens na atenção primária à saúde. Interface, Comun., Saúde, Educ. [Internet]. 2012 [acesso em 2015 ago 29]; 16(40). Disponível em: http://dx.doi.org/10.1590/S1414-32832012005000021.

4. Horta NC, Lage AM, Sena RR. Produção cientifica sobre políticas públicas direcionadas para jovens. Rev. enferm. UERJ [Internet]. 2009 [acesso em 2015 ago 14]; 17(4). Disponível em: http://www.facenf.uerj.br/v17n4/ v17n4a15.pdf.

5. Mafra MP. Ações em saúde para adolescentes nos serviços de atenção básica - o olhar do enfermeiro em um distrito sanitário [dissertação]. Curitiba (PR): Universidade Federal do Paraná; 2014.

6. Penso MA, Brasil KCTR, Arrais AR, Lordello SR. A relação entre saúde e escola: percepções dos profissionais que trabalham com adolescentes na atenção primária à saúde no Distrito Federal. Saúde Soc. [Internet]. 2013 [acesso em 2015set 15]; 22(2). Disponível em: http://dx.doi.org/10.1590/S0104-12902013000200023.

7. Marques JF, Queiroz MVO. Cuidado ao adolescente na atenção básica: necessidades dos usuários e sua relação com o serviço. Rev. gauch. enferm. [Internet]. 2012 [acesso em 2016 jun 17]; 33(3). Disponível em: http://dx.doi. org/10.1590/S1983-14472012000300009.

8. Núcleo de Educação em Saúde Coletiva (NESCON). Avaliação do impacto das ações do Programa de Saúde da Família na redução das internações hospitalares por condições sensíveis à atenção básica em adultos e idosos. Belo Horizonte: Universidade Federal de Minas Gerais - Faculdade de Medicina; Núcleo de Educação em Saúde Coletiva; 2009. 262 p. Relatório final. Disponível em: https://www.nescon.medicina.ufmg.br/biblioteca/ imagem/3261.pdf.

9. Nedel FB, Facchini LA, Martin M, Navarro A. Características da atenção básica associadas ao risco de internar por condições sensíveis à atenção primária: revisão sistemática da literatura. Epidemiol. Serv. Saúde. [Internet]. 2010 [acesso em 2016 jun10]; 19(1). Disponível em: http://dx.doi.org/10.5123/S1679-49742010000100008.

10. Ministério da Saúde (BR). Portaria ${ }^{\circ}$ 221, de 17 de abril de 2008. Publica em forma de anexo a lista brasileira de internações por condições sensíveis à atenção primária. Brasília: Ministério da Saúde. Secretaria de Atenção a Saúde; 2008 [acesso em 2016 fev18]. Disponível em: http://bvsms.saude.gov.br/bvs/saudelegis/sas/2008/ prt0221_17_04_2008.html.

11. Torres RL, Rehem TCMSB, Egry EY, Ciosak SI. The panorama of ambulatory care sensitive conditions in district of São Paulo. Rev. Esc. Enferm. USP [Internet]. 2011 [acesso em 2016 fev 23]; 45 Spec No. Disponível em: http:// dx.doi.org/10.1590/S0080-62342011000800004. 
sensíveis à atenção primária: a construção da lista brasileira como ferramenta para medir o desempenho do sistema de saúde (Projeto ICSAP - Brasil). Cad. saúde publica [Internet]. 2009 [acesso em 2016 maio 22]; 25(6). Disponível em: http://dx.doi.org/10.1590/S0102-311X2009000600016.

13. Medronho RA, Carvalho DM, Block KV, Roner LB, Werneck V, Guilherme L. Epidemiologia. São Paulo: Atheneu; 2006.

14. Melo MD. Internações por Condições Sensíveis à Atenção Primária em Guarulhos: um olhar da Enfermagem em Saúde Coletiva [dissertação]. São Paulo (SP): Escola de Enfermagem da Universidade de São Paulo; 2014.

15. Egry EY. Saúde coletiva: construindo um novo método em enfermagem. São Paulo: Î́cone; 1996.

16. Breilh J. Epidemiologia crítica: Ciência emancipadora e interculturalidade. Rio de Janeiro: Hucitec; 2006.

17. Estado do Paraná (PR). Instituto Paranaense de Desenvolvimento Econômico e Social (IPARDES). Caderno Estatístico Estado do Paraná - Julho. [Internet]. Paraná: IPARDES; 2016 [acesso em 2016 nov 05]. Disponível em: http://www.ipardes.gov.br/cadernos/MontaCadPdf1.php?Municipio=19.

18. Instituto Brasileiro de Geografia e Estatística (IBGE). Sinopse do Censo Demográfico 2010. [Internet]. Brasília: IBGE; 2010 [acesso em 2016 ago 09]. Disponível em: http://www.censo2010.ibge.gov.br/sinopse/index. php?uf $=41 \&$ dados $=26$.

19. Departamento de Informática do Sistema Único de Saúde (DATASUS). Cadastro Nacional de Estabelecimentos de Saúde (CNES). [Internet]. Brasília: DATASUS; 2011 [acesso em 2016 ma 04]. Disponível em: http://www2. datasus.gov.br/DATASUS/index.php?area=0204\&id=6906.

20. Santos LA, Oliveira VB, Caldeira AP. Internações por condições sensíveis à atenção primária entre crianças e adolescentes em Minas Gerais, 1999-2007. Rev. Bras. Saúde Mater. Infant. [Internet]. 2016 [acesso em 2016 set 10]; 16(2). Disponível em: http://dx.doi.org/10.1590/1806-93042016000200006.

21. Mendonça SS, Albuquerque EC. Perfil das internações por condições sensíveis à atenção primária em Pernambuco, 2008 a 2012. Epidemiol. Serv. Saúde [Internet]. 2014[acesso em 2016 jun 27]; 23(3). Disponível em: http://dx.doi.org/10.5123/S1679-49742014000300009.

22. Granda E, Breilh J. Investigação da Saúde na Sociedade: guia pedagógico sobre um novo enfoque do método epidemiológico. São Paulo: Cortez; 1989.

23. Resta DG. "Em relação ao sexo tudo é curioso": um modo de pensar a sexualidade de jovens na perspectiva da vulnerabilidade e do cuidado em saúde [tese]. Porto Alegre (RS): Universidade Federal do Rio Grande do Sul; 2012.

24. Cardoso CS, Pádua CM, Rodrigues-Júnior AA, Guimarães DA, Carvalho SF, Valentin RF et al. Contribuição das internações por condições sensíveis à atenção primária no perfil das admissões pelo sistema público de saúde. Rev Panam Salud Publica. [Internet]. 2013 [acesso em 2016 ago 23]; 34(4). Disponível em: https://www.scielosp. org/pdf/rpsp/2013.v34n4/227-234/pt.

25. Rehem TCMSB, Ciosak SI, Egry EY. Internações por condições sensíveis à atenção primária no hospital geral de uma microrregião de saúde do município de São Paulo, Brasil. Texto Contexto Enferm. [Internet]. 2012 [acesso em 2016 jun 13]; 21(3). Disponível em: http://dx.doi.org/10.1590/S0104-07072012000300007. 
26. Rodrigues-Bastos RM, Campos EMS, Ribeiro LC, Firmino RUR, Bustamante-Teixeira MT. Hospitalizations for primarycare sensitiveconditions in a Southern Brazilianmunicipality. Rev. Assoc. Med. Bras. [Internet]. 2013 [acesso em 2016 out 02]; 59(2). Disponível em: http://dx.doi.org/10.1016/j.ramb.2012.11.001.

27. Egry EY, Fonseca RMGS, Oliveira MAC. Ciência, Saúde Coletiva e Enfermagem: destacando as categorias gênero e geração na episteme da práxis. Rev. Bras. Enferm. [Internet]. 2013 [acesso 04 out 2016]; 66(nesp). Disponível: http://dx.doi.org/10.1590/S0034-71672013000700016.

28. Monteiro MOP, Costa MCO, Vieira GO, da Silva CAL. Fatores associados à ocorrência de sífilis em adolescentes do sexo masculino, feminino e gestantes de um Centro de Referência Municipal/CRM - DST/HIV/AIDS de Feira de Santana, Bahia. Adolesc. Saude. [Internet]. 2015 [acesso em 2016 set 14]; 12(3). Disponível em:http://www. adolescenciaesaude.com/detalhe_artigo.asp?id=520.

29. Duarte SJH, Ferreira SF, Santos NC. Desafios de enfermeiros da Estratégia Saúde da Família na implantação do Programa Saúde do Adolescente. Rev. Eletr. Enf. [Internet]. 2013 [acesso em 2016 jun 14]; 15(2). Disponível em: http://dx.doi.org/10.5216/ree.v15i2.18179.

30. Rehem TCMSB, Egry EY. Internações por Condições Sensíveis à Atenção Primária no Estado de São Paulo. Ciên Saúde Coletiva. [Internet]. 2011 [acesso em 2016 out 12]; 16(12). Disponível em: http://dx.doi.org/10.1590/ S1413-81232011001300024. 